Narrow Band Imaging (NBI) have failed to demonstrate reliable endoscopic signs of carcinoid which are often misdiagnosed as hyperplastic, adenomatous or neoplastic lesions, warranting histopathological diagnosis. Furthermore, microcarcinoids are usually an incidental diagnosis during routine gastric biopsy. Here we evaluate the use of Linked Colour Imaging (LCI); the latest Fujifilm post- processing digital technology for the endoscopic diagnosis of Type 1 GCTs.

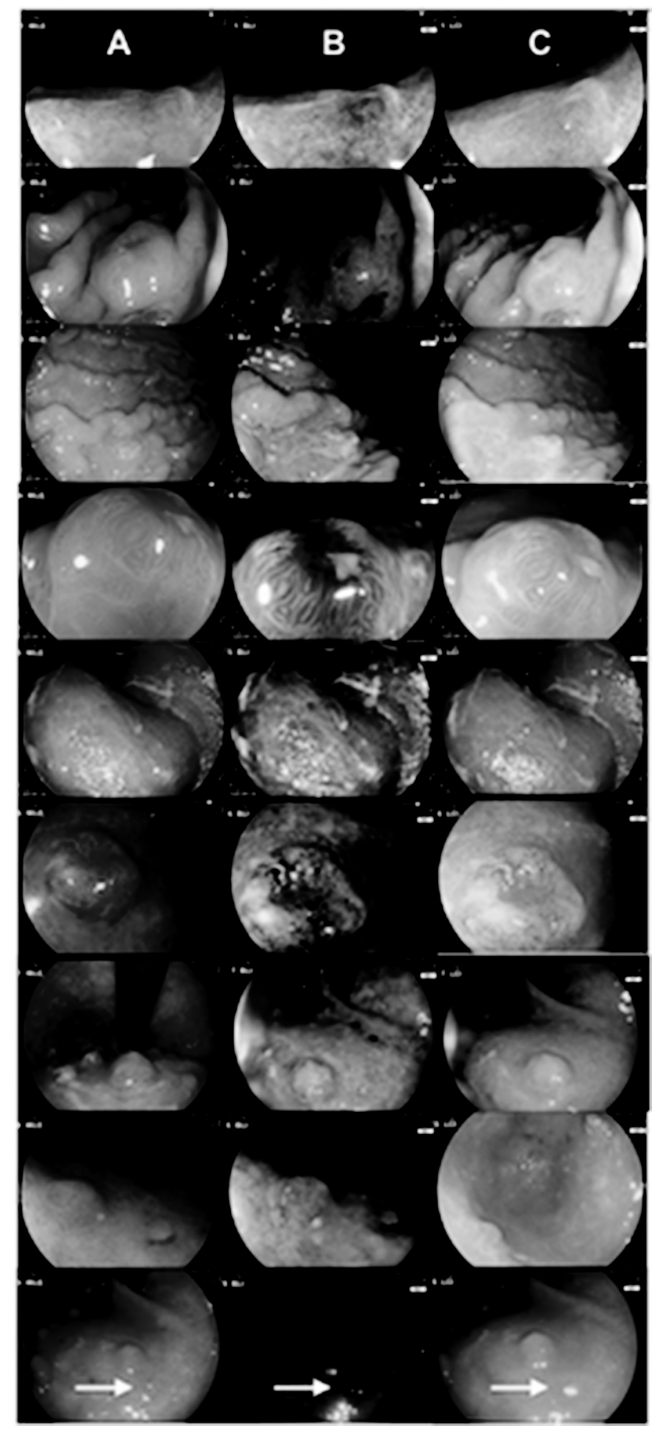

Abstract PTU-028 Figure 1 Type 1 GCT seen in WLE (A), BLI (B) and $\mathrm{LCl}(\mathrm{C})$

Methods Consecutive patients undergoing endoscopic surveillance of Type 1 GCTs were included. Patient baseline demographics were recorded. Endoscopic examination was performed using Fujifilm ELUXEO ${ }^{\mathrm{TM}}$ EG-760Z gastroscopes and simethicone/saline irrigation with imaging performed in the following sequence; WLE, blue laser imaging (BLI) and finally LCI. Lesion number, visibility using a known endoscopic scale (1-4; poor-excellent), endoscopic diagnosis were recorded for each imaging modality. Lesion demarcation and surface pattern features using LCI were recorded. High quality images of histopathologically confirmed Type 1 GCTs were selected for independent review by 2 further endoscopists blinded to histopathological diagnosis and inter-observer agreement calculated.

Results 3 patients (2 F), mean age 51.6 years were included. The total number of gastric lesions identified by WLE, BLI and LCI were 14,8 and 24 respectively. LCI identified an additional 10 and 16 gastric lesions compared to WLE and BLI respectively. Mean lesion size was $6.5(2-15) \mathrm{mm}$. Atrophic gastritis was confirmed histopathologically in all patients. Nine lesions with optimal image quality were selected for further review (Figure.1). Endoscopic features included, villous/ inflammatory surface pattern $(\mathrm{n}=9,100 \%)$, dense vasculature $(n=9,100 \%)$ and an amber hue $(n=9,100 \%)$. Diagnostic accuracy for Type 1 GCTs using WLE, BLI, and LCI were $22 \%, 22 \%$ and 100\% respectively. Median visibility of all lesions for both WLE and BLI were 2 (1-4) and 4 (3-4) using LCI. All lesions were well demarcated using LCI, 44\% with WLE and 22\% with BLI. Inter-observer agreement for the LCI diagnosis of Gastric NET was 100\%.

Conclusions LCI increases diagnostic yield and accuracy compared to both WLE and BLI and provides consistent endoscopic features;a novel feature over the challenges of prior imaging modalities. Lesion demarcation is clearer using LCI;an important factor to guide successful and complete endoscopic resection.

\section{PTU-029 ARE EXTRA-PANCREATIC MALIGNANCIES MORE PREVALENT IN PATIENTS WITH INTRADUCTAL PAPILLARY MUCINOUS NEOPLASM OF THE PANCREAS?}

${ }^{1}$ Trevor Tabone* ${ }^{2}{ }^{2}$ Kelvin Cortis, ${ }^{3}$ Neville Azzopardi. ${ }^{1}$ Department of Medicine, Mater Dei Hospital, Msida, Malta; ${ }^{2}$ Department of Radiology, Mater Dei Hospital, Msida, Malta; ${ }^{3}$ Department of Gastroenterology, Mater Dei Hospital, Msida, Malta

\subsection{6/gutjnl-2018-BSGAbstracts.314}

Introduction The association between the presence of an intraductal papillary mucinous neoplasm (IPMN) of the pancreas and the prevalence of extra-pancreatic malignancies (EPM) remains unclear. This is important with regards to determining suitable follow-up plans for IPMN patients. This single-centre, retrospective study aims to determine whether the prevalence of EPM is higher in IPMN patients as compared to the general Maltese population.

Methods A cohort of 175 patients with an incidental radiological diagnosis of IPMN on magnetic resonance imaging between 2010 and 2017 were recruited from a single, main centre in Malta. The prevalence of a previous history or synchronous diagnosis of EPM was recorded by reviewing electronic histopathology results of biopsies or resection specimens. EPM was defined as per ICD-10 (International Statistical Classification of Diseases and Related Health Problems) C00-80, thus excluding non-melanoma skin cancer and haematological malignancies. All EPMs were based on a tissue diagnosis. The prevalence of EPM was calculated and statistically compared with the lifetime prevalence of developing EPM (ICD-10, C00-C80) in the general Maltese population. Data regarding population demographics was obtained from the National Statistics Office and the National Cancer Platform.

Results 36 out of a total of 175 IPMN patients were found to have an EPM resulting in a prevalence of $20.57 \%$. The commonest malignancies were breast $30.6 \%(n=11)$, colorectal 
25.0\% $(n=9)$, and renal cell carcinoma 11.1\% $(n=4)$ respectively. The calculated lifetime prevalence (risk) of developing an EPM (Adjusted for Multiple Primaries - AMP method) in the general Maltese population is $19.5 \%$ (1 in 5). This was not found to be statistically significantly different when compared to the IPMN patient cohort $(\mathrm{p}=0.86)$.

Conclusions A previous history or synchronous histological diagnosis of EPM was not shown to be more prevalent in patients diagnosed with an IPMN of the pancreas, as compared to the general Maltese population. Given these findings, there is currently no rationale for undergoing further thorough investigations for an EPM in IPMN patients. The need for prospective, long-term follow-up studies in such patients is paramount to establish incidence rates for EPMs following an IPMN diagnosis.

\section{Small Bowel \& Nutrition}

\section{OWE-019 MECHANISMS OF CHEMOTHERAPY-INDUCED DIARRHOEA}

Stephanie French*, Andrea Davies, Munir Pirmohamed. University Of Liverpool, Liverpool, UK

\subsection{6/gutjnl-2018-BSGAbstracts.315}

Introduction Bcr-Abl inhibitors, such as bosutinib and imatinib, are predominantly used for treatment of chronic myeloid leukaemia. However, lower gastrointestinal toxicity, such as diarrhoea, is a prevalent adverse drug reaction (ADR). For example, bosutinib and imatinib cause diarrhoea in up to $90 \%$ and $50 \%$ of patients, respectively. ${ }^{1}$ This can decrease patient quality of life, treatment efficacy and in severe cases cause patient hospitalisation. We aim to elucidate the mechanism of $\mathrm{Bcr}-\mathrm{Abl}$ inhibitor-induced diarrhoea to help abrogate the aforementioned issues.

Methods Caco-2 cells (human colorectal cancer cells resembling small intestinal cells) were differentiated into monolayers of polarised enterocytes and utilised as an in vitro model. Cells were seeded into transwells and electrical resistance or flux of FITC-dextran (a fluorescently labelled polysaccharide) across the monolayer was measured to assess changes in paracellular permeability. Enteroids (small intestinal organoids) produced from male $\mathrm{BALB} / \mathrm{c}$ mice were used as an ex vivo model. Changes in permeability of enteroids were determined by leakage of injected FITC-dextran out of the enteroid. Changes in mRNA levels, protein levels and protein localization of tight junction components were studied using RTqPCR, immunoblotting and immunofluorescence, respectively. Drug-induced cell death was assessed by CellTitreGlo and Toxilight assays for Caco-2 cells and enteroids, respectively. Results were analysed by ANOVA and are representative of $\geq 3$ independent experiments.

Results $25 \mu \mathrm{M}$ bosutinib increased paracellular permeability of Caco-2 monolayers to ions and FITC-dextran (ANOVA, $\mathrm{p}<0.05$ ), whilst imatinib was less effective at inducing this change. $10 \mu \mathrm{M}$ bosutinib increased enteroid leakage (ANOVA, $\mathrm{p}<0.01$ ) but $10 \mu \mathrm{M}$ imatinib had no effect. All concentrations tested were sub-apoptotic.

In Caco-2 cells, bosutinib caused relocalization and decreased protein levels of intercellular junction proteins Ecadherin, Occludin and ZO-1. Bosutinib also transiently decreased mRNA levels of ZO-1 but not that of E-cadherin or Occludin. Imatinib did not alter mRNA levels, protein levels or localization of any of these proteins.

Endoplasmic reticulum (ER) stress is involved in intercellular junction degradation; ${ }^{2}$ therefore, we assessed whether Bcr$\mathrm{Abl}$ inhibitors could induce ER stress. However, no increase in ER stress markers BiP or CHOP were detected after bosutinib or imatinib treatment in Caco-2 cells.

Conclusions Decreased intestinal barrier integrity is likely an important factor in the aetiology of bosutinib-induced diarrhoea. This is potentially mediated by intercellular junction degradation. Understanding the mechanism by which Bcr-Abl inhibitors induce diarrhoea will aid in abrogation of diarrhoea ADRs.

\section{REFERENCES}

1. Br J Haematol. 2015;168:69.

2. Toxicol Appl Pharmacol. 2014;278:45.

\section{OWE-020 SURVIVAL AND CT DEFINED SARCOPENIA IN PATIENTS WITH INTESTINAL FAILURE ON HPN}

${ }^{1,2} \mathrm{SM}$ Oke* ${ }^{*}{ }^{1} \mathrm{~B}$ Rye, ${ }^{1,2} \mathrm{G}$ Malietzis, ${ }^{1} \mathrm{R}$ Baldwin, ${ }^{2} \mathrm{RA}$ Bottle, ${ }^{1,2} \mathrm{SM}$ Gabe, ${ }^{1} \mathrm{PFC}$ Lung. ${ }^{1} \mathrm{St}$ Mark's Hospital, Harrow, UK; ${ }^{2}$ Imperial College London, UK

\subsection{6/gutjnl-2018-BSGAbstracts.316}

Introduction Sarcopenia is recognised in patients with intestinal failure (IF) and has been associated with poorer survival in several chronic diseases. CT can measure sarcopenia through a L3 skeletal muscle index (LSMI).

We aim to evaluate the prevalence of sarcopenia in our IF population using LSMI, and evaluate the effect of home parenteral support (HPN) on LSMI and survival. Additionally, we aim to assess any association between LSMI, BMI and other anthropometric measurements.

Methods IF patients on HPN treated at St Mark's Hospital between 1/1/2006-1/10/2016 were identified from a prospectively maintained database. Patients were included if they were on HPN and had 2 CTs: the first $\leq 30$ days before start of HPN (pre-HPN); the second $\geq 100$ days from HPN start (post-HPN). Patient records were reviewed to obtain clinical and demographic information and date of death. Anthropometric measurements and BMI contemporaneous to CT scans were recorded.

Results 64 patients met inclusion criteria (M:F 1:1). 83\% of our cohort had LSMI below previously published thresholds for sarcopenia. Mean pre-HPN LSMI was $36.5 \pm 6.8 \mathrm{~cm}^{2} / \mathrm{m}^{2}$. Mean BMI pre-HPN was $22.1 \pm 4.8 \mathrm{~kg} / \mathrm{m}^{2}$. Both BMI $(\mathrm{p}<0.001)$ and LSMI $(\mathrm{p}=0.003)$ increased post-HPN. A positive correlation was seen between BMI and LSMI pre $(\mathrm{p}<0.001)$ and post-HPN $(\mathrm{p}=0.003)$. No correlation was seen between LSMI and anthropometric measurements pre-HPN $(\mathrm{p}=0.78)$ or post-HPN $(\mathrm{p}=0.96) .11(17 \%)$ patients died during the study period; a low LSMI pre-HPN was not a risk factor for mortality (HR $0.97 \mathrm{p}=0.55$ ).

Conclusions This study is the first to look at sarcopenia and survival using CT defined LSMI in the IF population. $83 \%$ of our cohort had a pre-HPN LSMI below previously published thresholds, yet we found no relationship between lower baseline LSMI and survival. This may reflect the heterogeneity of the prognoses of the IF population, or that parenteral nutrition itself affects survival. 